\title{
The impact of 2006 guidance on nutrition support, in clinical outcome measures
}

\author{
R. P. Vincent ${ }^{1}$, C. H. Goo ${ }^{2}$, P. F. Wu ${ }^{3}$ and C. W. Le Roux ${ }^{1}$ \\ Departments of ${ }^{1}$ Clinical Biochemistry, ${ }^{2}$ Dietetics and ${ }^{3}$ Pharmacy, King's College Hospital NHS Foundation Trust, \\ Denmark Hill, London SE5 9RS, UK
}

The 2006 National Institute for Health and Clinical Excellence (NICE) guideline 32 on nutrition support suggests that patients at risk of malnutrition should be screened on initial admission to hospital and where appropriate be referred to a dietician ${ }^{(1)}$. The aim of this audit was to compare our current parenteral nutrition (PN) prescribing practice for adult hospitalised patients, against the 2006 NICE guideline on nutrition support ${ }^{(1)}$. The audit focused on initial nutrition screening and early dietitian referral of patients with malnutrition or at risk of malnutrition. We investigated whether screening influenced outcome measures of PN administration such as: number of days on PN, total number of PN bags used per patient and length of hospital stay. This prospective audit was conducted over 5 months. Eighty patients were referred to the multi-disciplinary PN team, which consists of chemical pathologists, dietitians and pharmacists. Information was obtained from medical notes, electronic patient records, completed PN prescription charts, dietetic record cards and nursing care plans. Data was collected by the same two healthcare professionals throughout the audit, to ensure the data quality was not affected by investigator differences. The nutritional status of patients was determined based on the 2006 NICE guideline ${ }^{(1)}$ :

\begin{tabular}{ll}
\hline Patient demographics & \\
\hline Total number of patients & 80 \\
Mean age & 60 (SD 2) years \\
Gender: Male $(n)$ & $63(78.7 \%)$ \\
$\quad$ Female & $17(21.3 \%)$ \\
Clinical speciality $(n):$ & \\
$\quad$ Medical & $21(26.2 \%)$ \\
Surgical (elective) & $49(61.3 \%)$ \\
Surgical (non-elective) & $10(12.5 \%)$ \\
Wards $(n):$ Critical (ICU/HDU) & $34(42.5 \%)$ \\
$\quad$ General & $46(57.5 \%)$ \\
Nutritional assessment $(n)$ & \\
$\quad$ Nutritional screening on admission & $74(92.5 \%)$ \\
Patients at risk and malnourished & $51(63.7 \%)$ \\
Patients at risk of re-feeding syndrome & $22(27.5 \%)$ \\
Patients referred to dietitian & $39 / 51(76.5 \%)$ \\
\hline
\end{tabular}

The eighty patients were divided in to two groups as follows: Group A, screened on admission and referred to dietitian if malnourished or at risk of malnutrition as defined in 2006 NICE guideline ${ }^{(1)}$ prior to commencing on PN; Group B, patients not fulfilling one or more of the NICE criteria:

\begin{tabular}{|c|c|c|}
\hline & \multicolumn{2}{|c|}{ Group } \\
\hline & A & $\mathrm{B}$ \\
\hline Number of patients & 55 & 25 \\
\hline Patients malnourished or at risk $(n)$ & $29(53 \%)$ & $18(72 \%)$ \\
\hline Patients referred to dietitian $(n)$ & $29(100 \%)$ & $7(39 \%)$ \\
\hline Wards $(n)$ : Critical & $27(49 \%)$ & $7(28 \%)$ \\
\hline General & $28(51 \%)$ & $18(72 \%)$ \\
\hline $\begin{array}{l}\text { Number of days nil by mouth prior to PN } \\
\text { Clinical outcome measures }\end{array}$ & $2(\mathrm{SD} 1)$ & $4(\mathrm{SD} 2)$ \\
\hline PN withdrawal reasons $(n)$ : & $37(67 \%)$ & $18(72 \%)$ \\
\hline Tolerating sufficient enteral feeds & $8(15 \%)$ & $1(4 \%)$ \\
\hline Transferred to other hospitals/other reasons & $0(0 \%)$ & $2(8 \%)$ \\
\hline Suspected line sepsis & $10(18 \%)$ & $4(16 \%)$ \\
\hline \multicolumn{3}{|l|}{ Died or care withdrawn } \\
\hline Mean days duration on PN & $11(\mathrm{SD} 1)$ & $8(\mathrm{SD} 2)$ \\
\hline Mean number of PN bags per patient & $10(\mathrm{SD} 1)$ & $10(\mathrm{SD} 2)$ \\
\hline Mean days of hospital stay & $40(\mathrm{SD} 4)$ & $34(\mathrm{SD} 5)$ \\
\hline
\end{tabular}

The audit was a cross sectional study of practice. Patients whose care satisfied the NICE criteria for screening were more likely to be in critical care wards. However, there was no significant difference in any of the outcome measures between groups who satisfied or did not satisfy the 2006 NICE screening criteria.

1. NICE (2006) Clinical Guideline 32. Nutrition Support in Adults: oral nutrition support, enteral tube feeding and parenteral nutrition; London: NICE. 